\title{
Donner des consignes a un sosie et adopter un autre regard sur les possibilites de developpement des manieres d'agir au travail. Elements de reflexion a partir d'une intervention en sante au travail
}

\author{
Yvon Miossec*
}

\begin{abstract}
Résumé
Nous nous intéressons à une méthode de verbalisation et d'analyse de l'action : l'instruction au sosie. Notre analyse cherche à éclairer les usages que les professionnels peuvent faire de cette activité verbale et dialogique sur leur travail. Dans le cadre de la théorie historico culturelle en psychologie, il est possible de mettre en lumière les procédés actifs dans la transmission de consignes à un sosie. On expose des réalisations langagières dans le cadre d'une intervention en santé au travail qui montrent que l'analyse du travail est un moyen potentiel de transformation du travail

Mots clé: Analyse du travail, verbalisation, instruction au sosie, clinique de l'activité, développement du pouvoir d'agir, intervention en santé au travail.
\end{abstract}

\begin{abstract}
We are interested in a method of verbalisation and analysis of work : the instructions to a double. We clarify the uses that professionnals make of this dialogical method. In the context of historical-cultural theory in psychology, it is possible to highlight the active processes in the transmission of instructions to a double. We explain dialogues which arise from an an occupational health intervention. These dialogues show that work analysis is a potential way of work tranformation.

Keywords: Verbalization, work analysis, instructions to the double, activity clinic, extension of power to act, occupational health intervention.
\end{abstract}

\section{Introduction}

Cet article est consacré à la présentation de l'usage que des professionnels peuvent faire d'une technique particulière de verbalisation et d'analyse du travail : l'instruction au sosie ${ }^{1}$. Développée dans les années 70 dans le cadre de « communautés scientifiques »d'un genre nouveau associant travailleurs et psychologues du travail, la méthode de l'instruction au sosie vise à établir une coopération entre professionnels et chercheurs/ intervenants. La volonté d'établir de nouveaux rapports entre expérience du travail et psychologie académique (ODDONE et al., 1981) conduit à parler de co-analyse du travail. A partir des années 90, Clot a introduit la méthode dans la formation des psychologues en clinique du travail (CLOT, 1995, 1999, 2008). Avant d'intervenir dans les milieux de travail (son futur métier), chaque étudiant est invité à formaliser et élaborer sa propre expérience professionnelle. Il interroge alors pour lui-même et à propos de son propre travail les rapports entre activité et subjectivité. Ce faisant, il éprouve les rapports réciproques entre faire et connaître, mettre en mots et transformer (SCHELLER, 2003). Il examine les possibilités de faire de son expérience professionnelle vécue un objet d'analyse et un moyen de faire de nouvelles expériences (REILLE BAUDRIN, 2011). Dans nos propres travaux, nous mobilisons cette méthode d'auto-confrontation dans le cadre d'interventions en santé au travail. L'analyse du travail est alors regardée comme un moyen de développement du pouvoir d'agir des professionnels et de développement des métiers (MIOSSEC, 2011, MIOSSEC et al., 2014).

Dans cet article, nous poursuivons le travail de mise en lumière des processus psychologiques que le dispositif et sa méthode cherchent à activer et à soutenir. Les thèses de Vygotski sur le langage comme instrument d'organisation et de régulation de l'activité et la conceptualisation de l'activité de Leontiev reprises dans le travail d'Yves Clot constituent notre cadre théorique. Notre point de départ est le suivant : chaque sujet organise sa propre activité en définissant les buts de ses actions et se donnant des consignes $^{2}$ pour agir. L'instruction au sosie est une

\footnotetext{
*Endereço eletrônico: yvon.miossec@ lecnam.net
} 
Donner des consignes a un sosie et adopter un autre regard sur les possibilites de developpement des manieres d'agir au travail. Elements de reflexion a partir d'une intervention en sante au travail

technique qui permet de redécouvrir les buts que les professionnels cherchent à atteindre et les consignes qu'ils se donnent. Notre perspective est développementale. Aussi, nous regardons les effets que la connaissance de l'expérience et la capacité des sujets à l'utiliser pour retoucher l'expérience. Dans cet article, nous examinons spécifiquement les procédés techniques actifs dans l'instruction au sosie et les usages que des professionnels peuvent faire de ce " drôle de dialogue » dans le cadre d'une intervention en santé au travail.

\section{L'activite comme execution non consciente de consignes}

Parler du travail : une activité qui ne va pas de soi

Il faut partir d'un postulat qui heurte le sens commun et qui déroute souvent nos interlocuteurs (directions d'entreprises commanditaires d'intervention, collectifs de professionnels et sujets individuels engagés dans nos interventions) : le «parler du travail » ne va pas de soi. Le «travailler» n'existe pas, et encore moins spontanément, comme objet disponible à l'esprit et qu'il suffirait de mettre en mots ${ }^{3}$. Même si tout un chacun peut parler de son travail ${ }^{4}$, une discussion et une réflexion qui tiennent compte de la complexité de l'activité ordinaire ne sont pas possibles «naturellement », même si elles peuvent avoir lieu dans des contextes ouverts au dialogue. L'activité ordinaire de travail n'est pas transparente. Il est nécessaire de se doter d'instruments spécifiques pour y « accéder ». Pour pouvoir être discutée et réfléchie, elle doit être extraite du flux de son exécution et constituée en objet de pensée. C'est à dessein que nous écrivons les choses dans cet ordre chronologique (dire puis penser) puisqu'il s'agit pour nous de mettre en lumière la fonction du dialogue avec autrui sur son activité comme instrument du développement de la pensée sur cette même activité. La difficulté à constituer l'activité ordinaire en objet de discours et de pensée du fait de son caractère tacite a été travaillée en psychologie du travail. On s'appuiera ici sur les réflexions de Leplat à propos des compétences. Il écrit : "Certaines compétences sont difficiles à expliciter »; "Le sujet peut exécuter des tâches [...] qu'il ne peut exactement ${ }^{5}$ décrire par des mots » (1997, p.141). Leplat parle de « compétences incorporées » pour désigner «ce type de compétences qui s'expriment bien dans l'action, mais moins bien ou pas du tout dans le discours »(LEPLAT, 1997, p.141.). Alors que ces compétences sont facilement mobilisables dans l'action (" accessibles» dans le vocabulaire de Leplat, p.142), elles sont « très difficiles à verbaliser » (p.143). Faire son travail au quotidien (au plan pratique et au plan psychique), c'est en grande partie mobiliser son expérience, sans y penser. Ceci constitue un obstacle à l'analyse et au partage de l'expérience. Comme on va le voir, cet obstacle peut être travaillé. Les manières de faire, les gestes professionnels, les mobiles qui poussent le sujet à agir ne sont pas disponibles pour une verbalisation immédiate mais ils peuvent être décomposés et reconstitués en objet de pensée, ici via un discours adressé à autrui ${ }^{6}$. Cette (re)construction est possible car les « composantes automatisées » de l'activité n'ont pas toujours eu ce caractère non-conscient. Les habitudes, les routines, les automatismes, les « savoirs d'expérience » (LEPLAT, 1997, p.155) de l'activité ordinaire sont des acquis, résultat d'une expérience au cours de laquelle ils ont d'abord été conscients. C'est ce dont rend compte la théorie de l'activité de Leontiev qui distingue les composantes de l'activité. La distinction entre actions et opérations est ici centrale. L'action est « un processus soumis à un but conscient » et le sujet de l'action se représente le résultat qui doit être atteint (LEONTIEV, 1984, p.113). Ce qui n'est pas le cas des moyens qu'il mobilise pour atteindre ce but. Les opérations, moyens d'accomplissement de cette action existent, quant à elles, « sous la forme de pratiques automatisées » (LEONTIEV, 1976, p.296). Ainsi, celui qui travaille, conscient du but qu'il poursuit, atteint ce but en mobilisant des moyens, qui eux ne sont plus conscients. Mais, les opérations, non-conscientes, ont d'abord été des actions conscientes. Les procédés opératoires qui permettent l'exécution d'une action actuelle sont le fruit d'un apprentissage. Durant leur phase d'acquisition, les futures opérations ont elles aussi été des « processus visant à un but précis » (LEONTIEV, 1976, p.296). Leontiev donne l'exemple du tireur à la carabine. Celui-ci fait prendre à son corps une certaine position, amène le guidon du fusil en position strictement verticale, serre la crosse contre son épaule, retient sa respiration etc. Le tireur exercé accomplit tous ces gestes alors qu'il «n'a 
qu'un but dans sa conscience : toucher la cible » (LEONTIEV, 1976, p.296). Avant de maîtriser tous ces gestes et de les mobiliser sans qu'ils soient nécessaires de se les représenter, le tireur a d'abord appris à tenir correctement son fusil. Durant cette phase, la tenue du fusil était une action. Dans l'activité psychique de l'apprenti tireur, la tenue du fusil existe consciemment comme but à atteindre. Le tireur y pense, peut-on dire, et il se donne pour consigne d'agir pour atteindre ce but. Ensuite, chez le tireur averti, seul est présent à l'esprit le but «toucher la cible». La tenue du fusil est incorporée dans l'action « toucher la cible » dont elle est devenue un moyen. Et ce moyen est mobilisé sans y penser. Pour parler comme Leplat, Le tireur averti « ne saura plus dire, immédiatement et sans hésiter, comment on fait » pour tenir un fusil. Les « moments qui devaient absolument être conscientisés au début» (LEONTIEV, 1976, p.297) ont cessé de l'être. Mais et ceci nous intéresse particulièrement ici, ils peuvent à nouveau « être conscientisés » par lui (LEONTIEV, 1976, p.297) ${ }^{7}$.

Résumons-nous. Les activités ordinaires de travail sont notamment composées d'habitudes et de routines, procédés opératoires de l'action mobilisés « sans contrôle attentionnel » (LEPLAT, 2005). Il n'en a pas toujours été ainsi. Les opérations, aujourd'hui « automatisées » ont d'abord été l'objet d'un travail de conception et d'organisation. Maîtrisées, répétées tous les jours, comme c'est le cas dans l'exemple que nous allons exposer, elles sont ensuite exécutées sans y penser. Mais elles peuvent être redécouvertes pour parler comme Oddone.

L'origine et les formes de l'auto organisation de l'activité : définir des buts et se donner des consignes pour agir

Pour Leontiev, les actions sont les composantes essentielles des activités humaines (1984 p.113) et donc des activités de travail auxquelles nous nous intéressons. Nous allons rappeler la triple origine des buts avant de nous pencher sur la forme verbale de ces buts dans l'organisation de l'activité. Au travail, la définition de ces buts vient de plusieurs sources. Sous la forme de la tâche prescrite, l'organisation, relayée par la hiérarchie, fixe les buts à atteindre et est supposée attribuer des moyens pour y parvenir. Comme les sciences du travail l'ont établi de longue date, le travail n'est jamais réductible à l'exécution de la tâche prescrite devenu but à atteindre. Ainsi, l'activité (« ce qui est fait»), n'est pas réponse automatique aux prescriptions du milieu. Le but qui organise l'action ne s'origine pas seulement dans la tâche (la définition officielle de « ce qui est à faire »). En tant que sujet, le travailleur cherche à développer une expérience professionnelle productrice de sens (CLOT, 1995). Il cherche aussi à travailler de manière économique, à être efficient ${ }^{8}$ (CLOT, 1995). A la différence de l'automate, il cherche à avoir « la main » sur ce qu'il fait et à en faire quelque chose pour lui. Ainsi, ce sont aussi des buts personnels qui organisent son action. Enfin, le but de l'action se construit à partir d'une troisième source, celles des manières communes de prendre et de s'y prendre avec la tâche et avec l'organisation pour faire ce qui est à faire. Clot appelle genre professionnel cet « intercalaire social » $(1999, \mathrm{p}$. 34) « instrument social de l'action » (p. 31). Le genre professionnel sédimente les buts de l'action définis collectivement par ceux qui sont du même métier ${ }^{9}$. Engagés en première ligne dans les situations de travail (à la différence de ceux qui écrivent les prescriptions, eux-mêmes en première ligne dans leur propre situation), les collectifs de professionnels retravaillent la tâche sur la base d'évaluations de ce qui possible ou impossible, souhaitable ou à proscrire, juste ou déplacé, acceptable ou non. Cette troisième source des buts de l'action, sociale comme la tâche, se construit dans et par l'expérience ordinaire de " gens de terrain ». Ainsi, le but de l'action est-il toujours un compromis métastable entre le but explicite prescrit par l'organisation, le but implicite, validé collectivement et porté par le genre professionnel et le but singulier propre à chaque professionnel.

Le langage joue un rôle central dans le processus de la fixation et la réalisation des buts de l'action. Dans ses travaux sur les rapports entre pensée et langage, Vygotski propose une analyse des fonctions du langage égocentrique particulièrement utile ici. Le langage égocentrique désigne ces paroles prononcées par les enfants quand ils réalisent une activité pratique. Les énoncés de l'enfant ont initialement la forme d'un discours adressé à autrui alors même que l'enfant semble ne pas s'occuper de savoir si on l'écoute ni attendre de réponse. En fait, pour Vygotski, l'enfant s'adresse à lui-même. Ses paroles aux allures de commentaire de l'action remplissent en fait une fonction « intellectuelle ». L'enfant raisonne à haute voix. Il se parle à lui-même pour 
penser la situation et organiser son action dans la situation. Il se donne des consignes d'action ${ }^{10}$. L'étude du langage égocentrique, appelé à s'intérioriser et à devenir silencieux, met en lumière cette fonction $\mathrm{du}$ langage comme instrument de l'organisation des actions. Ainsi, les mots, d'abord prononcés à haute voix puis adressés à soi dans le silence du langage intérieur, sont des moyens pour "se communiquer à [soi]-même les caractéristiques de la situation, les fins qu'[on] doit s'assigner, les moyens qu'[on] doit se donner " (BROSSARD, 2004, p.46). Ils permettent au sujet d'analyser les situations dans lesquels il se trouve et de se donner des instructions pour agir et dépasser les obstacles qu'il rencontre. Ainsi, les mots possèdent «un pouvoir » sur les fonctions psychiques et sur le comportement (VYGOTSKI, 2004 , p. 233-234). Ils sont le «moyen de diriger volontairement » la pensée et la conduite (VYGOTSKI, 1997, p.267). Le sujet les utilise pour se donner des directives et des consignes et ainsi planifier, réguler, guider ses actions. Réfléchie grâce aux mots, l'action devient moins réactive, moins immédiate, moins directement conditionnée par la situation (VYGOTSKI, 1994, p.229). Elle devient action volontaire ${ }^{11}$. L'action est plus libre, plus fluide car elle bénéficie de la dynamique de la pensée (VYGOTSKI, 1994, p.226-227).

Ainsi, les buts qui organisent et planifient l'activité, appelés pour beaucoup d'entre eux à devenir moyens non conscients, ont existé comme objectif et comme consigne que le sujet se donne pour travailler. Vygotski le rappelle, cette autoorganisation de l'activité (analyse de la situation et planification de l'action) reproduit au plan intrapersonnel $^{12}$ un processus interpersonnel de transmission de consignes. On dirige son activité d'une manière similaire à la manière dont on a été dirigé et dont on dirige les autres ${ }^{13}$. Ce qui conduit Vygotski à comparer, de façon un peu provocatrice pour nous, l'action du sujet sur ses propres processus à l'action du contremaître qui prescrit une tâche à un subordonné (2004, p. 234).

Planifiées, les actions peuvent être être replanifiées, réorganisées. Dans le protocole de l'instruction au sosie qu'on va présenter, la réorganisation des processus internes de planification des actions est soutenue par leur extériorisation. Ce qui compte ici, c'est le mouvement non plus du langage vers la pensée mais celui qui va de la pensée organisatrice de l'activité à sa mise en mots. En quelque sorte, on fait suivre aux consignes organisatrices de l'action un chemin inverse à celui de leur intériorisation. Cette extériorisation est une double transformation. La transformation de la pensée en paroles oblige le sujet qui veut être audible à réemprunter les formes du langage social. Il doit aussi, seconde transformation, non plus utiliser le langage pour se donner des consignes à lui-même mais pour transmettre des « ordres » à autrui. Le passage d'un dialogue avec soi-même en dialogue avec autrui et la transformation des consignes qu'on s'adressait soi-même, sans y penser, en consignes pour autrui peut produire des effets sur les consignes initiales et modifier l'auto organisation du travail.

\section{L'instruction au sosie: une technique pour se distancier de ses actions et les constituer en objet d'elaboration}

Quand il conçoit la méthode de l'instruction au sosie, Oddone cherche à accéder à l'auto organisation de l'activité. Il constate en effet que chaque ouvrier développe une expérience propre alors que la tâche est strictement la même pour tous $(1984$, p.28). Lui aussi partait du constat de l'impossibilité d'une mise en mots directe de l'organisation de son activité par le professionnel. Comme il l'indique, les sujets ne sont pas conscients du plan programme ${ }^{14}$ mis en œuvre quand ils travaillent (ODDONE et BRIANTE 1981, p.55). Ou plutôt, ils ne le sont plus. Ce plan programme a bien été conçu et donc pensé. Il est le résultat de consignes explicites que le sujet se donnait lors des phases d'apprentissage. Désormais, il est exécuté sans y penser. Il s'agissait pour Oddone d'inventer une méthode pour « formaliser l'expérience informelle » afin de « la rendre accessible » $(1981$, p. 58). Elle consiste « à demander à chaque sujet de donner des instructions à un moi auxiliaire, à un sosie » (1981, p.55). Comme on va le voir, la méthode de l'instruction au sosie mobilise plusieurs procédés qui visent à accéder à l'ordinaire de l'activité (« le répétitif » du travail quotidien) en la constituant en objet de discours et de pensée et en s'en détachant.

On trouve également le terme de « sosie » sous la plume de Vygotski. Pour lui, la conscience fonctionne comme un « contact social avec soi- 
même » (2003, p. 91) grâce aux capacités proprement humaines de différenciation entre sujet pensant et objet de pensée. Vygotski parle du caractère double de la conscience. Pour lui, « la représentation d'un sosie est la représentation de la conscience la plus proche de la réalité » (2003, p. 91). Pour autant, cette capacité humaine de différenciation entre conception et exécution des actions ne s'active pas aisément. Une fois qu'elles fonctionnent ensemble, constituées comme en un bloc (SIMONET, 2011, p.137), les manières d'agir existent pour le sujet comme meilleur compromis pour faire ce qui est à faire.

$\mathrm{La}$ forme particulière de verbalisation proposé par la méthode de l'instruction au sosie a vocation à soutenir des processus de reconception des buts des actions par déconstruction/ reconstruction des modes opératoires qui les réalisent et des mobiles que les actions cherchent à réaliser. Il s'agit d'une méthode indirecte de réorganisation de l'activité professionnelle. Le professionnel accède aux consignes/ buts qui organisent son activité en les extériorisant, en les adressant à autrui. Il développe le contact social avec lui-même via un contact avec autrui. En cherchant à faire de son interlocuteur un double de lui-même, le professionnel réactive cette capacité pour lui-même. La " fabrication » d'un sosie externe soutient le développement de la différenciation, de l'altérité au plan interne. Au plan temporel, les processus à l'œuvre pour les professionnels qui se livrent à l'exercice s'inscrivent dans le temps présent et dans la durée. La communication à l'adresse d'autrui soutient dès sa réalisation une communication avec soi-même. Dans un essai sur la parole intérieure, le philosophe français Victor Egger avait cette formule : "Quand nous parlons à haute voix, la parole intérieure n'est pas pour cela absente » (cité par POUZOULET, 2012). Dès le temps de la transmission de consignes à un sosie, les professionnels peuvent imaginer d'autres buts organisateurs de leurs actions et d'autres consignes qu'ils pourraient se donner. Mais la technique mise surtout sur le travail dialogique et réflexif différé pour lequel le temps de l'instruction va constituer une ressource. Les consignes transmises à autrui pour en faire son sosie sont enregistrées sur un support audio. Ainsi, elles sont "fixées 》, accessibles à l'analyse lors de la réécoute de l'enregistrement. Les questions posées par le sosie peuvent être reprises par le sujet qui peut alors les mettre au service de ses propres questionnements (existants et renouvelés).

Ainsi, la technique du sosie est d'abord une technique clinique destinée à soutenir les processus psychologiques de développement du pouvoir d'agir. Elle permet aussi, ce à quoi nous nous employons dans cet article, de produire des connaissances sur les modalités de ce même développement.

Les procédés actifs dans le protocole de l'instruction au sosie

Plusieurs procédés sont actifs dans le protocole de l'instruction au sosie qui contribuent à la constitution du travail ordinaire comme objet d'élaboration et au détachement du sujet par rapport à ce qu'il fait. Plusieurs règles que le sosie se donne organisent le dialogue entre le professionnel et son « aspirant » sosie et permettent la mise en œuvre de ces procédés. Elles sont condensées dans la formule qui inaugure et guide le dialogue : "Suppose que je sois ton sosie demain dans une situation de travail précise (2) que tu vas m'indiquer. Dis-moi exactement (3) ce que je dois faire (1) et comment je dois le faire (4) pour faire comme toi. L'idée est que personne ne s'aperçoive de la substitution entre toi et moi (ton sosie) ».

Ces règles ${ }^{15}$, qui organisent le dialogue conduit par le psychologue/sosie, contribuent à désorganiser le cours et l'allure des « conversations ordinaires » sur le travail. L'instruction au sosie est, si l'on peut dire un procédé très réglé dont la fonction est de dérégler la parole et la pensée spontanées sur le travail. C'est la mise en œuvre de ces règles par le sosie qui donne au dialogue son caractère inédit et insolite. Elles contribuent à la production d'énoncés inattendus qui sont autant de " matières à penser » pour les professionnels.

La première de ces règles introduit une rupture avec les attendus d'une investigation de l'activité. Le sosie ne demande pas au professionnel quelles sont les consignes qu'il se donne à lui-même pour organiser son travail. Il lui demande de lui donner des consignes à lui, son sosie. Pour le professionnel, il ne s'agit pas de dire comment il fait mais de dire à un autre que lui comment il doit faire pour faire comme lui. Cette voie détournée : donner à autrui les consignes qu'on se donne à soi-même, constitue un moyen indirect, par extériorisation, pour permettre au sujet d'accéder aux consignes qu'il se donne sans y 
Donner des consignes a un sosie et adopter un autre regard sur les possibilites de developpement des manieres d'agir au travail. Elements de reflexion a partir d'une intervention en sante au travail

penser et les examiner. Elle «force » aussi le détachement du sujet vis à vis de ce qu'il fait.

La seconde règle consiste à interroger une séquence précise d'activité. Ici, c'est la focalisation sur un espace-temps délimité qui force le travail d'objectivation. Ce n'est pas l'activité en général qui est interrogée. Le sosie demande des instructions pour travailler à l'identique du sosie par exemple le lendemain à 14h. "A quel endroit, je vais me trouver?». Le professionnel est ainsi invité à décrire son lieu de travail, description toujours surprenante. "Qui sera là ? », «Par quoi faut-il commencer pour faire comme toi ? »Le sosie, animé par le projet irréalisable mais «pris avec sérieux » de récupérer des consignes qui lui permettraient d'agir à l'identique du professionnel, interroge l'ordinaire, le banal, le familier.

On pourrait qualifier la troisième règle de " règle d'or ": elle consiste pour le sosie à s'intéresser, sans relâche, aux détails. Il s'agit ici d'empêcher l'instructeur de "monter en généralité » car cela oriente les discours sur le travail vers le "déjà dit à vocation monologique » (CLOT, 2008, p. 210). Or, c'est le " pas encore dit à vocation dialogique » qui est la voie la plus sûre pour séparer « celui qui parle ou agit de ses fonctionnements habituels »(CLOT, 2008.). On pourrait presque parler de "dissection »d'une situation de travail strictement située. Ici encore, c'est le particulier qui est visé. Le sosie ne demande pas des consignes générales sur le travail de tous les jours, mais sur le travail spécifique du lendemain en particulier ${ }^{16}$ dans un créneau horaire nettement délimité. Il n'interroge pas les relations de travail avec les collègues en général mais avec tel ou tel en particulier. Il ne s'intéresse pas aux machines, aux clients, aux dossiers mais à telle machine, tel client, tel dossier etc. Cette « insistance » à questionner précisément les détails d'une situation permet de la dénaturaliser. Transmis à un sosie via des consignes pour la reproduire à l'identique, l'activité familière devient pour partie étrangère et parfois même étrange aux yeux du professionnel (CLOT, 1999, p.157). Pour répondre aux questions du sosie, comme «Comment je me déplace dans le bureau ? » ou «Comment je lis mes mails ? », questions pour lesquelles aucune réponse préparée n'est disponible à la conscience, le professionnel doit chercher «à se voir » en activité. Il doit se livrer à une « introspection », activité dans laquelle « l'esprit [...] se divise en objet et en sujet » (VYGOTSKI, 1999, p.274). Ceci nécessite et conduit à ce que le professionnel instaure une distance avec lui-même. Pris dans le cours du dialogue avec le sosie, il propose dans l'instant une réponse alors que concernant certaines questions elles demanderaient justement réflexion. Cette réponse, à la fois trop rapide et « un peu » réfléchie constitue une « objectivation momentanée de l'action » (BONNEMAIN et al., 2015, p.107). Comme tout dialogue, la transmission d'instructions au sosie est un dialogue inachevé. Mais ici ce trait est particulièrement marqué. Concernant sa forme, l'entretien au sosie a l'allure des dialogues quotidiens dans lesquels « l'énoncé n'est pas dissocié du monde de l'expérience »(BROSSARD, 2004 p. 75). Par sa fonction, il se rapproche de l'entretien de recherche. Le dialogue avec le sosie, orienté par les questions qu'il pose à vocation à constituer un recueil de données pour le professionnel qui donne les consignes. Ainsi, les réponses n'ont pas le statut de vérité sur l'activité mais de constitution d'un matériau verbal sur lequel il sera possible de revenir.

La spécification de l'objet du questionnement constitue une quatrième règle. Pour recueillir les consignes pour se conduire de la même manière que le professionnel, le sosie interroge la situation dans laquelle il va se trouver et l'activité qu'il va devoir réaliser. Le questionnement reste «opérationnel». Il porte sur le « quoi » et le «comment» et pas sur le « pourquoi ». Ici encore, le projet est indirect et mise sur le différé. Un questionnement sur le « pourquoi » peut conduire le professionnel à argumenter le bien-fondé de ses manières d'agir alors qu'il s'agit de redécouvrir ces manières d'agir et de les problématiser. Se justifier auprès d'autrui (le sosie et le groupe de pairs dans le cadre d'une intervention) est une activité qui peut rapidement s'avérer défensive. Ce sont de tout autres affects que la méthode cherche à provoquer. Il s'agit toujours de créer l'étonnement, source de la pensée $^{17}$. Les questions sur les dimensions opérationnelles du travail ordinaire fabriquent un obstacle inattendu : la difficulté à répondre à des questions d'apparence simple crée un effet de surprise. Donner des instructions sur ces « détails généralement non thématisés de l'activité » (KOSTULKI, 2011, p.81) permet de produire un 
discours extérieur sur les techniques d'action (le quoi et le comment) qui ne manqueront pas, au plan intérieur cette fois, d'ouvrir des questions sur le « pourquoi ». Ici encore, la constitution des actions comme objet de discours au service d'un dialogue inachevé produit des effets de différenciation pour le professionnel. Les actions sont, en quelque sorte, détachées du sujet (CLOT, 1999, p.156). Elles « s'extérioris[ent], ouvrant d'autres possibilités de rapport au réel » (CLOT, 1999, p.156).

\section{L'instruction au sosie dans le cadre d'une intervention en sante au travail}

On voudrait maintenant rendre visibles la mise en œuvre de ces procédés techniques d'objectivation de l'action et de séparation du sujet de ce qu'il fait à partir de matériaux empiriques issus d'une intervention en santé au travail. L'intervention réalisée répondait à la commande d'un établissement public de formation professionnelle. La commande négociée avec cette institution portait sur le développement des ressources psychosociales comme alternative aux programmes classiques de lutte contre les risques psychosociaux (MIOSSEC, 2011). Ainsi, le travail de recherche/intervention, piloté par un comité mixte direction générale et syndicats, portait sur le développement $\mathrm{du}$ pouvoir d'agir des professionnels de cet établissement. La recherche s'est concentrée sur le travail des cadres. Deux métiers ont fait l'objet d'un travail approfondi: celui de directeur de région et celui de cadre en charge de l'organisation des actions de formation. Les matériaux cliniques mobilisés ici sont issus du travail avec les directeurs de région qui se sont portés volontaires pour participer à la recherche ${ }^{18}$. Il est composé d'un ensemble de traces de réalisations langagières (KOSTULSKI, 2011) que nous avons nous-même retranscrit ${ }^{19}$. Ainsi, nous allons proposer une analyse du contenu de l'instruction au sosie d'une directrice, Madame A, en regardant particulièrement l'activité du sosie. Nous chercherons à rendre compte des effets produits par ce type de verbalisation en nous appuyant sur le commentaire fait par Madame A., quelques semaines plus tard et des discussions qui ont eu lieu avec ses pairs directeurs dans le groupe d'analyse métier (GRAM) que nous avons mis en place. Nous montrerons comment l'analyse détaillée et la discussion de séquences bien délimitées de travail font entrer les buts dans une zone de développement potentiel (CLOT, 1995, p.35-36; 1999, p.159).

Mais, d'abord, une brève présentation du métier de directrice qu'occupe madame A. Madame A. est cadre dirigeant dans la fonction publique, dans le domaine de la formation professionnelle. Elle travaille dans une structure nationale présente sur l'ensemble du territoire, chacune de ces antennes régionales employant entre 15 et 100 personnes dont une majorité de cadres $^{20}$. Elle-même dirige une structure qui emploie quarante personnes. Elle s'est portée volontaire pour participer à la recherche sur le métier de directeur, recherche pour laquelle nous avons constitué un groupe national de directeurs régionaux. Ce groupe d'une dizaine de directeurs de ces structures de formation professionnelle s'est réuni une quinzaine de fois pendant deux ans, chaque directeur faisant le déplacement à Paris. A l'exception des séances de mise en place du dispositif et des séances de préparation des comités de pilotage, chaque séance était consacrée à l'analyse de situations de travail avec la technique de l'instruction au sosie. Les directeurs choisissaient des séquences de travail significatives et l'un d'entre eux se proposait de donner des instructions au sosie pour un « remplacement » dans cette séquence de travail. Deux psychologues du travail/ chercheurs, dont nous-même animions le travail du groupe.

Plusieurs séquences de travail ont ainsi pu être travaillées: l'animation d'une réunion d'équipe, la participation à une réunions institutionnelle, l'évaluation d'un collaborateur, le travail avec les adjoints dans un comité de direction etc ${ }^{21}$. L'activité de travail que Madame A. a choisi de transmettre au sosie est une tâche quotidienne. Elle consiste pour les directeurs à signer de nombreux documents externes (à destination des commanditaires des formations, des fournisseurs, des formateurs etc.) et de documents internes (dans les relations avec le siège national). La signature valide la conformité des actes réalisés par l'ensemble des agents. Elle engage l'institution. Il y a peu de prescription pour cette tâche, si ce n'est le respect d'un ensemble de règles de délégation de signatures. Les directeurs y consacrent chaque jour entre 30 et 60 minutes. Il s'agit donc pour les directeurs d'une activité qui par sa fréquence peut être qualifiée de routinière. Elle est désignée par les directeurs comme activité de signature des parapheurs (les parapheurs étant 
Donner des consignes a un sosie et adopter un autre regard sur les possibilites de developpement des manieres d'agir au travail. Elements de reflexion a partir d'une intervention en sante au travail

les classeurs regroupant les documents à signer).

Comme les autres réunion de travail directeurs/ psychologues du travail, la séance sur la signature des parapheurs est organisée en deux temps (réunion 1):

- Madame A. va transmettre des consignes au sosie pendant une quarantaine de minutes. Pendant ce dialogue, les autres directeurs écoutent et se préparent à discuter entre eux de l'activité de signature,

- s'ensuit un temps de discussion entre pairs sur la base de l'instruction au sosie (environ quatre vingt dix minutes).

L'ensemble de la réunion est enregistré sur un support audio. Les enregistrements de l'instruction au sosie et de la discussion qui suit (dans notre jargon, le retour à chaud) sont adressées au directeur qui a donné des instructions. Ses collègues, quant à eux, reçoivent l'enregistrement de la discussion à laquelle ils ont participé. Chacun est invité à écouter les enregistrements reçus.

La séance de travail suivante (réunion 2 : six semaines après dans ce cas) est consacrée à un nouveau retour (à froid, cette fois dans notre jargon) sur l'instruction au sosie. Le professionnel est invité à commenter son travail d'écoute et d'analyse et de livrer au groupe "l'état de sa réflexion ». Une tâche spécifique à réaliser entre la réunion 1 et la réunion 2 est prescrite au professionnel qui a donné des instructions au sosie : il doit écouter le dialogue et doit produire une analyse. Rappelons-le, le matériau sonore qui fixe le dialogue entre le sosie et le professionnel n'a pas ici «valeur de vérité » sur l'activité, encore moins de vérité définitive.

Il fixe la réponse faite dans l'instant, dans un contexte particulier, à un aspirant sosie qui pose des questions imprévisibles. Ainsi, le professionnel peut disposer d'une trace durable pour réfléchir à ce qui été dit, y revenir, rectifier, en un mot pour pouvoir s'expliquer avec les descriptions qu'il a donné de son travail en transmettant des consignes au sosie.

Un dialogue insolite

Le premier dialogue qu'on va lire reprend les dix premières minutes de l'échange entre Madame A. et son futur sosie. Avec ce premier verbatim, on souhaite donner une vue d'ensemble du type d'échange mis en cuvre par cette technique de verbalisation de l'activité. On interrogera les effets de la transmission de consignes au sosie en nous appuyant sur le commentaire oral produit six semaines plus tard.

1.SOSIE : Avant toute chose, est-ce qu'on peut se tutoyer le temps de cette instruction?

2.DIRECTRICE : On va essayer (rire)

3.S : On va essayer. Donc, imagine que je te remplace demain dans ta situation de travail. Je vais te demander de me donner des consignes pour que je fasse comme tu fais, que j'opère comme tu opères. Avec cet objectif que personne ne doive se rendre compte de l'échange, de notre substitution

4.D : D'accord

5.S : Il y a un enjeu fort, c'est ce qui fait que je vais poser les questions les plus précises possibles pour que je puisse te remplacer demain

6.D : D'accord

7.S : Demain je te remplace dans quelle situation? Parce que on va pas pouvoir faire toute la journée

8.D : Donc, je te propose de me remplacer au moment de la signature des parapheurs. D'autant plus que moi j'ai donné une habitude, c'est que les parapheurs soient déposés le soir. Tu n'as pas besoin d'aller les chercher. L'assistance les dépose sur $\mathrm{ma}^{22}$ table de travail en face de mon bureau

9.S : La veille?

10.D : Le soir même. Avant qu'elle parte, vers $4 \mathrm{~h}$ et demie $5 \mathrm{~h}$, soit elle me les dépose sur mon bureau, soit je sais qu'ils sont déposés dans son bureau tous à un endroit. Et que je vais avoir la possibilité d'aller les chercher pour les signer. Ça, c'est si j'ai eu du monde dans mon bureau. Mais la plupart du temps, les parapheurs sont déposés sur une table ronde, ovale dans mon bureau

11.S : Dans mon bureau. Alors pour que j'y vois clair ... donc demain je suis dans mon bureau. A quelle heure pour commencer à travailler sur les parapheurs?

12.D : A partir de 5 heures et demie, 6 heures

13.S : 5 heures et demie ou six heures plutôt ?

14.D : (Rire) Alors moi

15.S : Donc moi

16.D : Toi (rire)

17.S : (Rire) C'est moi qui opère, il faut que tu me dises précisément

18.D : Tu pourras commencer à les signer finalement qu'à partir de 18 heures. Avant, l'emploi du temps ne le permet pas 
19.S : C'est ça, demain, je sais que j'ai un emploi du temps qui va pas me permettre de m'y coller, quoi, à cette tâche avant 18 heures

20.D : Voilà

21.S : Alors, 18 heures, euh je suis dans mon bureau après une journée de travail

22.D : Oui, totalement. Après une journée de travail?

23.S : Moi j'ai besoin de savoir, en fin de journée de travail, je suis comment? Je suis dans quel état d'esprit?

24.D : Ben, tu es, si t'es comme moi (rire)

25.S : C'est le but (rire)

26.D : Tu es très, tu es du soir, en pleine activité le soir, tu es comment dire ? euh attentif le soir, ça c'est la première chose. Et tu es tranquille. C'est à dire, il n'y a plus de réunions à venir, voilà normalement moins de collaborateurs dans la délégation; il reste éventuellement les deux adjoints, éventuellement un ou deux cadres, il y a une certaine tranquillité

27.S : Donc, je suis dans mon bureau, l'esprit plutôt tranquille

28.D : Oui

29.S : Pour me coller. Tu vois, j'ai tendance à dire pour me coller à cette tâche

30.D : Oui

31.S : Mais c'est une façon de dire les choses, comment tu dirais, toi, quand tu ?

32.D : Non, moi je dirais, pour examiner les parapheurs qu'on m'a mis sur mon bureau et pour les signer

33.S : Examiner, je vais examiner des parapheurs

34.D : Voilà et c'est à dire, les parapheurs d'abord correspondent à des services, c'est à dire que moi je sais d'où vient le parapheur parce que j'ai fait faire des fiches navettes sur les parapheurs donc je sais quel est la personne qui a mis dans le parapheur, quel est l'émetteur du courrier, du document à me faire signer ou valider 35.S : Ou la la! Bon, voilà. Je suis perdu

36.D : Tu es perdu?

37.S : Je suis perdu (rire). Alors, je suis, voilà, je suis, l'état d'esprit tranquille, je vais donc examiner, hein, c'est le terme

38.D : Oui

39.S : Examiner les parapheurs. Je sens que j'ai une organisation de ces parapheurs. Est-ce que dans mon bureau, l'esprit tranquille...D'ailleurs, j'ai mon bureau qui est ouvert, qui est fermé, comment il organisé mon bureau ? je suis derrière mon bureau?

40.D Non parce que en fait dans ton bureau tu as le bureau où tu travailles, où tu as ton ordinateur, tes dossiers. Devant, il y a une table ovale où sont déposés les parapheurs

41.S : Ah !

42.D : Donc, tu te déplaces, tu te lèves, tu te déplaces, tu prends ton thé avec toi (rire) et tu poses ton thé et tu commences à regarder les parapheurs. Voilà

43.S : Donc, j'ai, il faut que j'adopte ce rituel ?

44.D : Oui, oui. Un peu cette cassure entre le rythme de travail de la journée, le dernier coup de téléphone et à ce moment-là, tu te dis : « Ah, c'est le moment d'aller regarder les parapheurs ", tu te lèves, tu vas sur ta table de travail en face

45.S : Ok

46.D : Normalement ils sont bien classés en fonction de la provenance. Normalement

47.S : Dis-moi maintenant ce que je trouve sur ma table ovale, que j'arrive à visualiser. Tu peux me décrire un peu?

48.D : Ouais, il y a des parapheurs qui sont, comment dire ? proviennent des différents services ou des différents valideurs des contenus de ces parapheurs

49.S : Lesquels?

50.D : Alors, les différents valideurs c'est les trois chefs de service, le DFi (directeur des finances) et le DF (directeur de la formation) puisqu'il y a des choses visées par le DF avant d'arriver chez moi; il y a des choses qui sont seulement visés par les chefs de service et puis il y a des choses qui sont visées par le $\mathrm{DFi}$, tout ce qui est comptable notamment; tout ce qui est lettre de missions, courriers aux commanditaires de formation, ça a suivi un circuit services, DF. Enfin, il y a différents circuits qui font que normalement un courrier, quand il arrive chez moi, il a été élaboré par une personne comment dire ? validé par elle et supervisé par le chef de service, voire visé par le cadre de formation d'ailleurs ou le chef de service et supervisé par le DF ou le DFi

51.S : J'ai peut-être mal compris mais j'ai l'impression que $\mathrm{j}$ 'ai alors trois, cinq classeurs

52.D : Il peut y en avoir cinq, enfin, cinq types

53.S : Combien j'en ai ?

54.D : Ah mais il y en a bien plus

55.S : Ah non mais, parce que tu m'as dit DF, DFi, trois chefs de services ça fait cinq pour moi

56.D : Cinq types

57.S : Mais il y en a d'autres?

58.D : J'ai pas réussi à faire en sorte, tu n'as pas réussi à faire en sorte que, par service, les courriers soient remis ensemble dans un même parapheur. 
Donner des consignes a un sosie et adopter un autre regard sur les possibilites de developpement des manieres d'agir au travail. Elements de reflexion a partir d'une intervention en sante au travail

Des fois, tu ouvres un parapheur, il n'y a qu'une pièce à signer. Donc ce qui fait qu'il y a un mur impressionnant de parapheurs mais, je dirais, ils se réunissent en différents types d'origine

59.S : Je viens de comprendre que je n'ai pas toujours le même mur de parapheurs sur ma table ovale, c'est ça?

60.D : Voilà, c'est jamais le même mur

61.S : Ah ouais, d'accord. Et les parapheurs arrivent, tu m'as dit à $16 \mathrm{~h} 30$ qu'ils arrivaient vers $16 \mathrm{~h} 30$

62.D : 16h30, $17 \mathrm{~h}$. Ils arrivent. Ils sont déposés sur ma table

63.S : Ou alors il faut que j'aille les chercher s'ils sont pas déposés. Ils arrivent d'un bloc, c'est ça ? La secrétaire

64.D : Oui, elle manipule des tas

65.S : Et éventuellement quand je vois le tas, enfin le mur, je jette un œil dessus ? Je me dis quelque chose sur «Tiens, aujourd'hui le mur est épais» ou pas du tout?

66.D : Oui, ben je le prends comme un constat, tu le prends comme un constat, ça dépend des jours. Mais voilà...

67.S : Ça m'agace, ça m'agace pas ?

68.D : Non, ça m'agace pas. C'est un temps consacré à ça dans ta journée, donc...

69.S : Peu importe la quantité de parapheurs que j'ai, ça ne me...

70.D : Non

Une transmission « impossible »

Comme on peut le lire, le sosie déploie les différents procédés qu'on a présentés. Elles donnent lieu à des formes spécifiques de réalisation langagière qui ouvrent la voie aux processus psychologiques de mise à distance d'avec ses actions en situation et d'avec soi-même. Que peut-on voir ? Comme l'indique Clot, « la transmission de consignes susceptibles de permettre un remplacement effectif est une mission, par définition, impossible ». (1999, p.155). Il n'empêche, le sosie s'y essaye (5). Il « joue le jeu ». Il fait « comme si » cela était possible. Il n'est pas du métier, il ne connaît rien de la situation, il n'a pas l'expérience de la professionnelle, mais il s'autorise, se distinguant nettement de l'adoption d'une posture de neutralité, à imaginer la situation et à y projeter son activité. Il ne cherche pas tant à soutenir le professionnel dans son effort de description qu'à récupérer les informations dont il a besoin pour le remplacer. Ainsi, il s'attelle à une mise en mots pour $l u^{23}$. Ce qui le conduit à « entraver le déroulement des opérations » (CLOT, 1999, p.156) que le professionnel exposerait s'il s'exprimait spontanément. Le sosie assume une position d'altérité. Il regarde la situation de son point de vue. On pourrait presque parler d'un entretien directif et peu compréhensif ${ }^{24}$. Il faut le préciser ici, le sosie ne cherche pas à établir les correspondances entre l'expérience professionnelle $\mathrm{du}$ sujet et des catégories scientifiques. Le dialogue de l'instruction au sosie réalise un échange entre les catégories empiriques du professionnel et celle de son aspirant sosie. Le sosie assume une position authentique d'ignorance de l'activité ordinaire d'autrui. Il cherche à récupérer des consignes en questionnant l'expérience du professionnel. Pour ce faire, il imagine la situation en mobilisant davantage ses propres expériences professionnelles que son expérience scientifique. Avant de savoir ce qu'il va devoir faire, il a besoin de savoir où il va se trouver et à quel moment l'action va se dérouler. Il a besoin de savoir et il le demande (23). Face à cette tâche impossible, il est perdu. Et là encore, il le dit (35). Pendant le temps qu'il joue le rôle du sosie, adoptant une posture qui tranche nettement avec celle qu'il adopte avant et après ce " jeu dialogique ", l'intervenant s'autorise à avoir un avis évaluatif sur une situation que pourtant il ne connaît pas. Il s'agit sans doute d'une activité un peu pénible, il faut " s'y coller » alors même que c'est la fin de journée (29 à 32).

L'obstination méthodologique du sosie à faire valoir ses préoccupations de remplaçant, l'insistance qui pourrait paraître déplacée ${ }^{25}$, " obligent » Madame A. à voir sa situation et son activité d'un autre point de vue que le sien. Ceci contribue à produire des effets de rupture. Les évidences sont heurtées. La confrontation du scénario proposé par le professionnel avec le scénario imaginé par le sosie fait dérailler ce qui va de soi pour Madame A., qui accomplit cette activité « automatiquement ». Ceci contribue à dénaturaliser la chaîne opératoire qu'elle met en œuvre dans cette activité routinière. A certain moment et ce point sera particulièrement net dans l'extrait que nous présentons ensuite, elle doit même se mettre à la place du sosie qui pose des 
questions qui n'en sont pas pour elle ou n'en sont plus. En empêchant la description qui lui viendrait naturellement à l'esprit, le sosie "force » le détachement de Madame A. de sa situation ordinaire de travail et des actions qu'elle y réalise. Ce faisant, le sosie construit une possibilité pour Madame A. de se déporter en amont des choix d'organisation de l'action qu'elle a opérés, parfois de manière un peu résignée. Le dialogue avec le sosie la détache de ses schèmes d'action usuels (CLOT, 1999, p.157). Les scénarios alternatifs imaginaires proposés par le sosie entretiennent l'idée que d'autres manières de faire sont possibles. Et cette idée constitue une force motrice pour la pensée du professionnel. Il ne s'agit pas bien sûr pour elle d'imaginer faire comme le sosie qui n'est qu'un interlocuteur artificiel. Mais le dialogue avec le sosie réouvre la voie d'un dialogue avec elle-même entre ce qu'elle fait et ce qu'elle pourrait - peut-être - faire.

«Au travers de mes réponses, je me suis posé plein de questions »

Lors de la réunion suivante du Groupe d'analyse du métier de directeur, Madame A. a produit un commentaire oral en soutenant son propos de notes manuscrites produites pendant l'écoute de l'enregistrement et peut-être retravaillées en vue de l'« exposé $»^{26}$. Y figure l'énoncé qu'on a repris en titre de ce paragraphe : «Au travers de mes réponses je me suis posé plein de questions ». Alors qu'elle a répondu à la prescription de se focaliser sur ce qui dans le dialogue avec le sosie a été source d'étonnement, elle a d'abord développé l'idée de son manque de clarté. Madame A. s'étonne de sa difficulté à être claire quand il s'agit de donner des consignes à autrui sur une activité quotidienne, répétée, «bien huilée », pourrait-on écrire. Il est probable que le commentaire de Madame A. est habité par l'idéal de clarté résumé par Boileau : « Ce qui se conçoit bien s'énonce clairement/ et les mots pour le dire arrivent aisément». Comme le sosie, elle «a joué le jeu » et elle pensait qu'elle saurait être plus explicite. Cette difficulté, vécue comme un peu déplaisante, constitue néanmoins une ressource pour l'élaboration en tant qu'elle conduit le sujet à poursuivre sa réflexion. Ainsi, la séquence choisie ne consiste pas seulement à signer et à examiner des documents $(8,32)$. «Il faudrait dire vérifier, superviser, contrôler ... La signature n'est que la fin du truc. En fait c'est tout un processus ». On a ici affaire à une nouvelle objectivation de l'activité de signature, activité plus complexe qu'elle ne l'énonçait. Ce qui a le plus étonné Madame A., « ce qu'[elle] s'est dit quand [elle] s'est écoutée » pour reprendre son expression, c'est le décalage entre ses réponses au sosie et la réalité. «J'ai dit : la secrétaire les dépose (10). C'est pas vrai. Dans $70 \%$ des cas, je suis obligée d'aller les chercher $»$. " J'ai dit : ils viennent par service (46). En fait c'est pas vrai, ils sont mélangés ». " Pareil, vous m'avez demandé quel était mon état d'esprit (23), j'ai donné des éléments qui peuvent laisser paraître que je suis tranquille (26) parce que c'est le soir et que j'ai fini de faire le reste, je prends un thé...J'ai dit que c'était un temps très maîtrisé, très organisé etc. Bon ben voilà c'est pas ça, en fonction du volume, j'espère que la dedans il n'y a pas trop de trucs, j'espère que je vais pas avoir trop de questions etc ... ». Ce décalage, les réponses « non conformes " à la réalité, la récurrence qu'elle relève dans son discours de l'adverbe « normalement », le fait qu'elle n'a pas «vraiment dit les mots qui [la] taraudent en permanence » la conduisent à qualifier la séquence quotidienne de signatures des parapheurs comme une épreuve. Elle voudrait moins « subir» pour reprendre ses mots. "Si j'arrive à avoir de telles difficultés encore et une telle angoisse vis à vis de ça au moment où je signe, je me suis dit : qu'est-ce que je fais pour que les choses deviennent plus faciles ? ». L'activité de Madame A. consiste notamment à organiser le travail des autres. Elle s'y emploie : elle donne aux autres des habitudes (8), elle a mis en place des fiches navettes (34). Elle trouve que « ce n'est pas forcément réussi » et elle saisit cette occasion pour se demander s'il elle pourrait procéder autrement, prendre de nouvelles initiatives. Ainsi, son activité de prescription peut être réinterrogée. Les buts qu'elle se fixe et les consignes qu'elle se donne peuvent être requestionnées.

\section{Une occasion manquée}

La poursuite du dialogue avec le sosie va lui permettre d'ouvrir une voie de reconception de son action par redéfinition des buts. Comme pour le premier verbatim, nous exposons d'abord le dialogue avec le sosie. Ensuite, nous reprenons des extraits de son commentaire produit à partir de l'écoute de l'enregistrement ${ }^{27}$. Puis, nous proposons une analyse de ce qui a été opérant, lui a permis de « voir » son activité (la constituer 
Donner des consignes a un sosie et adopter un autre regard sur les possibilites de developpement des manieres d'agir au travail. Elements de reflexion a partir d'une intervention en sante au travail

comme objet de pensée) en s'en détachant et d'imaginer des modes opératoires alternatifs.

La passation de consignes au sosie a conduit le dialogue à porter sur un « événement/ incident » ordinaire. Précisons les choses du côté des règles de fonctionnement de son travail. Madame A. a deux adjoints. L'un d'eux, directeur de la formation (DF) encadre le travail des cadres de formation qui eux-mêmes recrutent les formateurs $^{28}$ et établissent les conditions de leur intervention et de leur rémunération. C'est la directrice qui, in fine, signe les contrats d'embauche des formateurs. Il existe deux tarifs de base (tarifs B et C) et un tarif plus élevé (tarif A). Recruter un formateur au tarif A constitue une dérogation à la règle. Dans un contexte général de restrictions budgétaires, la direction générale demande aux directeurs de limiter strictement le recours à ce tarif qui doit rester exceptionnel. On va suivre le dialogue entre le sosie et la directrice.

101. SOSIE : Bon, qu'est-ce que je découvre ? A quoi je peux m'attendre?

102. DIRECTRICE : Et bien la plupart du temps tu découvres quand même des choses qui sont très récurrentes : des lettres de mission qui normalement ont été visées par le DF (Directeur de la Formation) et celui-ci doit avoir vérifié que toutes les règles concernant les formateurs, que leur niveau de tarification a bien été respecté

103. S : Dis-moi comment...Je vérifie ? Mon activité consiste à faire quoi ? A signer ?

104. D : A signer en ayant un œil sur deux trois choses très simples. Le tarif, si c'est du C ou du B, tu te poses pas trop de questions, tu fais confiance au DF

105. S : Je signe, c'est ça ?

106. D : Oui tu signes. Si c'est du tarif A (le ton de la voix est celui de quelqu'un qui rappelle une règle) tu dois trouver à côté une fiche où le cadre de formation justifie pourquoi il a pris un tarif supérieur et cette fiche doit avoir été visée par le $\mathrm{DF} / \mathrm{C}$ a veut dire que le DF se porte garant du fait qu'il accepte/ Ça veut dire que les arguments que le cadre lui a donnés sont nécessaires et suffisants pour qu'on déroge à un montant de tarification habituelle. Sur la page de gauche, il y a une fiche et celle-ci te montre que le processus de dérogation au niveau tarification a bien été respecté et que le DF est parfaitement au courant 107. S : C'est toujours de la dérogation?
108. D : C'est de la dérogation parce qu'on nous demande le plus souvent d'utiliser les tarifs $\mathrm{C}$ ou $\mathrm{B}$, le tarif $\mathrm{A}$ c'est vraiment quand on fait appel à un formateur qui a de l'expertise. Faut qu'on nous l'explique

109. S : Alors, dis-moi/ A ce moment-là, si je te comprends bien, je dois pas signer comme ça

110. D : Faut regarder

111. S : Alors dis-moi ce qu'il faut que je regarde

112. D : Tu regardes le formulaire qui a été établi par le cadre

113. S : Et qu'est-ce que je vais chercher là-dedans ?

114. D : Tu regardes les arguments, tu regardes d'abord si le DF l'a bien signé ça veut dire que déjà

115. S : Ah, il peut arriver qu'il ne le signe pas?

116. D : Il peut arriver qu'il le loupe

117. S : Ah. Qu'il le loupe?

118. D : Oui, s'il a fait vite un soir

119. S : Ou qu'il ne le signe pas?

120. D : Ou qu'il ne le signe pas effectivement. Moi, je pars toujours du principe qu'il l'a loupé (rire) mais il peut l'avoir...

121. S : Donc si c'est pas signé je considère que le DF...

122. D : A visé le parapheur mais n'a pas été assez attentif

123. S : N'a pas été assez attentif. Bon

Lors des tours de parole suivants, non retranscrits $\mathrm{ici}^{29}$, le sosie demande à la directrice quel type d'argumentaire il trouvera. La directrice rappelle à nouveau l'importance du visa du DF. Si le DF a signé, elle peut s'appuyer sur le travail de son adjoint. Se repose alors la question des demandes de dérogation non signées par son adjoint.

123. S : A quel moment je décide que c'est recevable ou que ça n'est pas recevable?

124. D : Alors, très honnêtement, si le DF a signé, je n'approfondis pas

125. S : Je signe?

126. D : Oui. Parfois, je cherche des documents qui sont en dessous. Pour essayer de comprendre ce qu'il y a dans le...Enfin, c'est pas de la signature toujours automatique, c'est pas...Tu cherches le document dont t'as besoin. La fiche dont on parle là. Si elle est pas là, elle a été glissée en dessous, tiens. Ça peut arriver. Normalement on demande qu'elle soit à gauche. Mais parfois elle est glissée en dessous.

127. S : Je vais chercher... Donc, quand le DF a 
signé, j'ai compris. Mais quand il s'est loupé?

128. D : Quand il s'est loupé, et bien ...deux options. Soit il est encore dans son bureau. Tu te lèves (ton très expressif, fait un petit sifflement) tac tac tac/ Tu vas voir le DF qui a la plupart du temps sa porte ouverte et tu lui dis

129. S : Je frappe quand même?

130. D : Oui, quand même, tu frappes parce que souvent il a la tête sur son truc. Donc, tu frappes, c'est mieux

131. S : Comme toi, je fais comme toi

132. D : Tu frappes. (elle prononce son nom) «

Philippe » Tu lui demandes si tu peux lui parler d'un parapheur ou bien tu lui dis : "J'ai une question. Là, il y a une lettre de mission tarif $\mathrm{A}$ mais vous n'avez pas signé Philippe, vous n'avez pas signé l'attestation. C'est volontaire, c'est un oubli ? ». Si c'est volontaire : "Pourquoi ? ». Il me dit : «Ah, non c'est un oubli ». Hop, tu lui fais signer. Tu lui fais régulariser. En live. Tu le fais et c'est bon

134. S : Quand j'ai à me lever, je prends le parapheur, je lui mets sous le nez

135. D : Oui

136. S : Enfin, je lui demande si je peux. Enfin, je prends les formes avec Philippe

137. D : Bien sûr, tu lui balances pas comme ça sur le bureau

138. S : Je m'entends bien avec Philippe ? Il y a une relation?

139. D : Ouais y a une relation.

140. S : Mais il me voit venir quand il me voit rentrer comme ça à cette heure ci ?

141. D : Oui

142. S : Comment il m'accueille parce qu'il a aussi ?

143. D : Il est en train de faire ses mails, de faire son travail

144. S : Il m'accueille ? Il me voit venir ? Il s'en doute ? Il anticipe ?

145. D : Il s'en doute parce qu'il voit que tu as un parapheur dans les mains. Il t'accueille poliment parce que c'est quelqu'un de poli et...C'est quelqu'un, tu peux y aller, il ne manifeste jamais de manifestation d'agacement parce que... Si t'as une question, il va te répondre très volontiers parce que... Il va dire « J'ai oublié. Excusez-moi » 146. S : Ah voilà "J'ai oublié, excusez-moi ». Est-ce que c'est une formule que $\mathrm{j}$ 'attends, que je considère comme minimale parce que $\mathrm{j}$ 'ai dû me lever quand même ?

147. D : Oui, tu l'attends. Puis, tu repars, tu dis « merci. Bon Ok merci »
148. S : Alors avant de dire : «Ok merci ». Quand il me dit : "Ah oui j'ai oublié ou je ne sais pas quelle formule ». Est-ce que je lui soumets le document et je le laisse faire ? Comment je me tiens, je reste debout face à lui ?

149. D : Tu restes debout. Tu lui dis : «Ecoutez, voilà. Pouvez-vous regarder ce document ? » Soit il le prend tout de suite parce que c'est un oubli, soit tu vois qu'il n'est pas prêt à le faire parce qu'il était en train de taper. Tu lui déposes ouvert en lui disant « Regardez plus tard. Vous me redirez. Vous me le ramènerez ».

150. S : Est-ce que je peux m'autoriser à engager une discussion sur le fond?

151. D : S'il y a une question de fond bien sûr tu peux t'autoriser. Après, oui, il faut ressentir s'il va être disponible. Il est disponible physiquement. Il va jamais dire «Pff ! ». On va pas...mais s'il est en train de faire un mail, je te conseille de lui dire : « J'aimerais avoir une discussion parce que moi ça me pose question et tout de suite ou plus tard, je suis là dans mon bureau. On peut en parler quoi »

Le sosie questionne la directrice pour savoir quels documents il va trouver dans le parapheur. L'échange va porter sur un document en particulier : la lettre de mission pour recruter les formateurs. A partir du tour de parole 108, le sosie comprend qu'il devra porter une attention particulière aux recrutements avec des tarifs dérogatoires. Il poursuit en s'intéressant à ce qu'il doit faire concrètement. Il découvre l'importance $\mathrm{du}$ visa du directeur de la formation. Si l'adjoint a signé, ce que normalement il devrait faire (102, $114,124)$ la directrice et donc le sosie aura simplement à confirmer cette signature en toute confiance (104). Mais la directrice le prévient : il arrive que le document ne soit pas visé par le DF. S'il remplaçait la directrice, il trouverait parfois des demandes de dérogations non visées par lui. Le sosie demande alors ce qu'il doit faire face à cette situation ce qui conduit Madame A. a donner des instructions sur le mode opératoire à suivre pour récupérer la signature (128 à 151). Il devra aller voir le directeur de la formation dans son bureau et la directrice indique au sosie comment va se dérouler l'échange avec son adjoint. Selon elle, l'absence de signature relève d'un manque d'attention qui conduit l'adjoint à " louper » parfois la signature. Elle décrit ensuite un échange particulièrement bref. Le sosie trouve probablement sa manière d'agir un peu expéditive. En tout cas, il ne se voit pas opérer de la sorte. Il 
Donner des consignes a un sosie et adopter un autre regard sur les possibilites de developpement des manieres d'agir au travail. Elements de reflexion a partir d'une intervention en sante au travail

ne se sent pas équipé pour exécuter le plan programme qui organise l'action de Madame A. Du coup, il résiste à la description, il dit vouloir mettre les formes $(129,136)$. Pour réussir à se projeter dans la situation, il a besoin de connaître la tonalité des relations de travail avec son collaborateur (138) et savoir comment ce dernier va l'accueillir (140, 144). Ici encore, le déroulement automatique des opérations est entravé. Les obstacles posés par le sosie, les scénarios alternatifs qu'il propose font dérailler l'enchaînement automatique des opérations et permettent à Madame A. d'objectiver son activité, de s'en détacher et de pouvoir la regarder.

En commentant son écoute du dialogue avec le sosie, Madame A. reviendra avec prudence sur le travail avec son adjoint. Faire confiance en son adjoint ne va pas de soi. "Je dis que je fais confiance (voir 104). Enfin, je le dis ». S'ouvre pour elle un questionnement qu'elle dira souhaiter poursuivre. «Il ne faut pas globaliser. Ce n'est pas : « je ne fais pas confiance ou je fais confiance », il $\mathrm{y}$ a plein de choses sur lesquelles je fais totalement confiance ». Elle se demande : " Qu'est-ce que je fais pour que ce moment (de la signature) soit plus facile ? », « Comment avoir moins de ressentiment du fait de la non adéquation du comportement de mes collaborateurs avec ce que j'exige d'eux ? ». Elle réénonce les conditions dans lesquelles elle souhaiterait se trouver «Si les responsabilités sont bien exercées, moi, je dois pouvoir signer les yeux fermés en téléphonant à quelqu'un d'autre ». Le déroulement de la séquence dans lequel elle vient recueillir la «signature loupée » la questionne. Cette fois, ce sont ses « schèmes relationnels » 30 qui sont constitués en objet d'élaboration. Elle évalue le déroulement de l'échange et se demande s'il est possible de s'essayer à faire autrement pour re développer la qualité de la collaboration avec son adjoint ? «Ma façon de répondre m'a obligée à me dire : 'Je suis très affirmative là' mais ça soulève des questions sur la méthode de travail » « Est-ce que c'est le meilleur moment d'aller voir quelqu'un quand il fait autre chose ? " Vous m'avez dit : 'Si je vais voir le $\mathrm{DF}$, il va m'accueillir comment ?'. J'ai dit qu'il est très poli, que ça ne va pas l'agacer (soupir). Qu'est-ce que j'en sais ? Peut-être que ça l'agace en fait que je vienne, que c'est pas le moment, que je le dérange. Peut-être que lui, il préférerait que je lui en parle à un autre moment ? Mais est-ce que je lui ai posé la question ? Jamais ». Le sosie, parce qu'il n'est pas engagé dans la situation de travail et qu'il ne voit pas les choses comme elle, fait l'hypothèse qu'une discussion de fond aurait son utilité (50). Malgré sa réponse (51), les consignes qu'elle donne avant la question du sosie (49) laissent à penser qu'en situation ordinaire, il est peu probable qu'elle engage une discussion avec son adjoint. Le scénario proposé par le sosie constitue un point de comparaison pour la directrice qui remet alors en question l'argument classique du manque de temps. «Parce qu'on est un peu dans...des fois on dit, on est un peu dans la lessiveuse, dans l'essoreuse. On n'a pas le temps de reposer, de discuter, de prendre le temps autour d'une situation qui pose question. C'est pas seulement les questions de la situation mais de dire finalement avec celui qui est là, qui l'a écrit, qui l'a supervisé d'échanger sur ce que ça génère. On n'a pas le temps, enfin je devrais pas dire ça, on prend pas le temps de ça ». La question des échanges informels rebondira dans la discussion entre directeurs qui rappelleront que la question des dérogations est source de tensions vives dans l'institution. Tous ces dialogues (avec le sosie, avec ses pairs, avec elle-même) conduiront Madame A. à décider de s'y prendre autrement avec le DF. Elle restera discrète sur l'expérience peut être tâtonnante à laquelle elle s'est livrée pour transformer la relation professionnelle avec son adjoint mais elle indiquera en percevoir des effets dans une prévention nouvelle de son adjoint vis à vis d'elle quand il soumet à la signature des dossiers difficiles.

Du dialogue avec le sosie au dialogue entre pairs

Ce dernier point sera abordé rapidement dans le cadre de cet article mais le dispositif est conçu pour être utile à chaque participant du groupe. Chaque directeur signe chaque jour de nombreux parapheurs ${ }^{31}$. Quand les membres du groupe de pairs écoutent leur collègue donner des instructions au sosie, ils s'intéressent aux consignes données par l'instructeur au sosie mais ils imaginent aussi les réponses qu'eux-mêmes auraient apporté à ses questions. Objet d'écoute, l'instruction au sosie du collègue peut devenir pour chaque professionnel un moyen pour explorer sa propre organisation et ses propres routines. Ainsi, la focalisation sur le mode opératoire de l'un 
d'entre eux permet à chacun de constituer son propre mode opératoire en objet de réflexion. Tout en étant occupé à une activité très différente (donner des consignes au sosie/ écouter les consignes du collègues), avec des moyens différents (parler, écouter), les pairs du groupe peuvent « faire fonctionner » le dialogue instructeur/ sosie comme moyen pour penser, de manière encore différente ici, leur propre organisation du travail. La formule d'un des professionnels, qui cherchent à comprendre la démarche dans laquelle ils sont engagés, permet de résumer les choses : "Chacun s'entend. Ensuite, quand on retourne à notre travail, on regarde ». En écoutant Madame A., chaque directeur " met en relation la situation de son collègue avec les situations qu'il traverse lui-même » et peut constater que des « difficultés qu'[il] croyait personnelles [peuvent] se révéler en tant qu'embûches de métier auquel chacun se confronte » (KOSTULSKI, 2011). Les ficelles de métier peuvent être partagées (KOSTULSKI, 2011), chacun peut réfléchir à ses arbitrages et éventuellement en imaginer de nouveaux. Les consignes transmises au sosie par un des directeurs peuvent servir d' " étalon provisoire » qui provoque la discussion. Ce sera le cas à propos de la fonction du dialogue dans le travail avec les adjoints. Une discussion aura lieu sur le thème de ce qui sera nommé "échanges informels ». L'instruction au sosie de Madame A. laisse à voir que l'obtention de la signature semble l'emporter sur la discussion avec son adjoint. Y réagissant un de ses collègues soumettra à la discussion un arbitrage alternatif : «Dans nos métiers, on est beaucoup dans le formalisme. Or, moi je crois beaucoup aux échanges informels et le soir c'est souvent le moment où on peut faire ça justement. La question que tu te poses 'Est-ce que je vais le déranger ou pas le déranger', je ne me la pose pas. Quand j'ai besoin d'échanger, j'y vais et on prend le temps. Et tant pis pour le parapheur. Il attendra un jour de plus ». Ainsi, l'instauration de la confiance que Madame A. qualifiera parfois de question psychologique peut aussi être posée comme question technique ${ }^{32}$. On peut aussi le constater, les différences $^{33}$ qui sont discutées ne sont pas seulement des différences entre les personnes. Chez les directeurs, il y des écoles : par exemple entre ceux qui signent le soir et ceux qui signent au " fil de l'eau ». Les conflits de critères peuvent être discutés : signer le soir dans le calme/ signer dans la journée pour pouvoir consulter en direct ceux qui soumettent un document à la signature, pour «avoir les agents sous la main ». Pour certains directeurs la signature au fil de l'eau constitue même une opportunité pour " circuler dans les bureaux ». On voudrait terminer ce court commentaire sur les discussions entre pairs en rappelant que dans ces discussions la fonction du psychologue/chercheur est de soutenir l'expression des variantes, des différences dans les manières de procéder. S'occuper des détails continue de s'avérer fructueux. Le débat à ici porté sur les post it que les directeurs laissent dans les documents renvoyés à ceux qui les ont rédigés ou supervisés. Quelle formule utiliser pour entretenir les relations de confiance avec les agents et les adjoints. Peuton écrire " m'en parler»? Ou cela risque-t-il de braquer les collaborateurs. «Pour en parler » ou « Merci de m'en parler » sont-elles des formules plus adéquates et plus efficaces?

\section{Conclusion}

Dans cet article, nous avons présenté l'instruction au sosie, l'une des deux méthodes d'analyse du travail mobilisées en clinique de l'activité. Dans une perspective clinique, les professionnels ne sont pas, dans un premier temps du moins, des « informateurs » pour les experts en charge d'organiser le travail ou de prendre en charge la prévention des risques psychosociaux. Le dispositif vise à soutenir un « travail sur le travail » dont la finalité est le développement des ressources psychologiques et sociales mobilisables par les professionnels. Nous avons cherché à mettre en lumière l'usage développemental que les professionnels peuvent en faire dans le cadre d'un travail collectif entre pairs organisé sur la base de cette méthode. Ainsi, nous avons éclairé les processus que ce type de verbalisation peut soutenir et leurs fonctions de réorganisation de l'activité. Il nous faut aussi signaler que le développement de l'activité via l'instruction à un sosie ceci n'est pas systématique. C'est sans doute cette prévention à l'égard de tout irénisme qui a conduit Clot à préférer parler de zone potentielle (et non pas proximale) de développement (1995, p.36). On peut énumérer des obstacles spécifiques. Ils sont à replacer dans le champ plus large des difficultés de développement du pouvoir d'agir au travail (Ministère du travail, 2011). D'abord, il faut indiquer que le passage de l'analyse à la transformation peut se trouver durablement bloqué quand les interprétions existantes de la situation 
Donner des consignes a un sosie et adopter un autre regard sur les possibilites de developpement des manieres d'agir au travail. Elements de reflexion a partir d'une intervention en sante au travail

remplissent une fonction défensive et maintiennent les dimensions de l'activité qui font souffrir à l'écart du dialogue et de l'élaboration (DEJOURS, 1993). Ensuite, l'imagination d'autres actions possibles dans le contexte d'un travail d'analyse, source d'énergie, ne se traduit pas systématiquement en réorganisation effective des actions. Le retour dans la situation ordinaire fait passer la réorganisation imaginée à l'« épreuve des faits ». Quand le professionnel se donne de nouvelles consignes, la mise en œuvre de l'action replanifiée rencontre des obstacles et des résistances auxquels il n'avait pas pensé. Dans le meilleur des cas, il sera davantage équipé pour aborder avec une plus grande disponibilité et poursuivra le mouvement de reconception de ses actions. Mais, les nouveaux buts imaginés peuvent aussi ne pas trouver de voies de réalisation. Troisième point : il est aussi nécessaire de souligner la fonction du travail collectif dont on a donné ici un trop court aperçu. C'est une ressource centrale pour le développement individuel $\mathrm{du}$ rapport à la tâche, aux collègues, à la hiérarchie. Le dispositif accorde une place centrale à l'hétérogénéité des manières d'agir. Les professionnels du groupe cherchent ensemble des solutions aux obstacles qu'ils rencontrent sans chercher à définir the one best way taylorienne. Il ne vise pas à uniformiser les manières de faire (le général). Il travaille sur le métier en faisant l'inventaire, par nature inachevé, des cas particuliers ${ }^{34}$. Ce faisant, les professionnels font reculer les limites des actions possibles en enrichissant le stock générique des gestes de métier (CLOT, 1999). Vérifier entre collègues qu'il y a plusieurs manières de faire la même chose constitue une source potentielle de développement de la plasticité professionnelle de chacun. Une dernière prévention s'impose : mettre l'accent sur les ressources individuelles et collectives du travail ne doit pas occulter que la qualité de l'organisation du travail constitue une dimension centrale pour la santé au travail. Le milieu de travail est d'abord défini par l'organisation formelle qui définit les tâches, fixe les normes, d'attribue les moyens ${ }^{35}$.

Quand les conditions le permettent, et l'intervenant peut agir en ce sens, le travail collectif d'analyse doit aussi contribuer à retoucher l'organisation formelle : les règles, les procédures, les outils (BONNEFOND, 2015). C'est l'objet du travail avec le comité de pilotage qui peut déboucher sur l'instauration d'un travail renouvelé entre ceux qui conçoivent les prescriptions et ceux qui font le travail sur le terrain.

Il existe toujours un risque de « fétichisation » des méthodes. En ce qui concerne l'instruction au sosie, il est important de ne pas regarder cette technique en termes de «tout ou rien ». Après avoir énoncé plusieurs règles de prudence, il faut conclure en rappelant les « bénéfices » potentiels des interventions en santé au travail outillées par l'instruction au sosie. L'analyse et la verbalisation du travail via cette technique peut soutenir un processus de développement de l'activité du travail dont on a mis ici en lumière quelque moments. Dans un cadre favorable à la mise en question des situations ordinaires de travail, la confrontation au point de vue d'un «soi-disant» sosie et le dialogue entre pairs peut mettre en mouvement les « idées arrêtées » sur les manières d'agir au travail. Le recours à la posture du sosie rend possible une formalisation singulière et inachevée du travail. Par la médiation de différents différentes réalisations langagières (instruction au sosie, dialogues entre collègues, commentaires) prises dans plusieurs types de dialogues (avec les autres et avec soi), les professionnels accèdent à l'activité existante qui est mise en tension ${ }^{36}$ avec d'autres activités possibles. C'est sans doute pour ces raisons que les professionnels s'investissent souvent de manière intense dans ce type d'investigations de leur activité et d'échanges entre pairs.

\section{Notes}

1. Avec les auto confrontations vidéo, l'instruction au sosie est une des deux méthodes utilisées en clinique de l'activité.

2. Nous tenons instructions, consignes, ordres pour synonymes.

3. La question des rapports entre expérience et connaissance est un problème classique de la psychologie. Les formulations de Moscovici à propos des représentations sociales nous semblent transposables en psychologie du travail à propos de l'activité. Les hommes, écrit-il, «ne raisonnent pas sur [le savoir commun] et ne peuvent [le] placer en face d'eux comme un objet ». Ils ne peuvent pas «l'analyser dans ses contenus en se plaçant à 
distance pour l'observer, sans s'y impliquer » (MOSCOVICI, 2013, p.105).

4. Les discours immédiats, les « prêts à dire » sur le travail peuvent constituer des obstacles à l'élaboration. Souvent, la parole spontanée consiste en une description du travail comme exécution des buts fixés par la tâche et les professionnels énoncent la tâche, ce qui est à faire. Elle peut aussi consister en l'expression de «ressentis », se constituer en discours de plainte (Foli). D'où la nécessité de mettre en place dispositifs d'intervention qui offrent aux professionnels la possibilité de parler de l'activité et de la réfléchir : ce qu'ils font et comment ils le font. Dans une perspective clinique développementale, le discours sur le travail est sollicité pour devenir un moyen d'agir sur le travail.

5. De notre point de vue, « exactement » traduit une euphémisation du problème de la verbalisation de l'activité.

6. La conscience (le psychisme, dit Vygotski) est cette capacité humaine à «sélectionner des éléments stables de réalité au sein du mouvement universel » et de «constitue [r] des îlots de sécurité dans le flux héraclitéen » (1999, p. 167). Ainsi, il est possible d'introduire un point d'arrêt dans le flux des activités psychiques et pratiques de l'exercice professionnel. L'expérience vécue peut être objectivée et cette objectivation peut contribuer à la transformer en moyen pour faire d'autres expériences (VYGOTSKI, 2003, p. 78 ; CLOT, 2008, p.79).

7. Peut-être pas de manière aussi évidente que ne le laisse parfois à penser le texte de Leontiev.

8. Dans son modèle de l'activité, Clot (1995) a d'abord mobilisé les concepts de sens (but de l'action/ mobile de l'activité) et d'efficience (but de l'action/ moyens opérationnels). Par la suite, il a développé la notion de pouvoir d'agir qui « unifie sans les éliminer les trois concepts qui [...] servaient à penser les développements possibles ou impossibles de l'action, l'efficacité rapportée à l'efficience et au sens » (CLOT, 2008, p. 102, note). Le pouvoir d'agir «augmente ou diminue en fonction de l'alternance fonctionnelle entre le sens et l'efficience de l'action où se joue le dynamisme de l'activité » ( p.12).
9. Qui eux-mêmes prolongent l'expérience de ceux qui les ont précédés dans la constitution de cultures professionnelles vivantes.

10. Alors qu'il doit récupérer un objet placer sur une armoire, il va dire « il faut monter sur la chaise », « je vais prendre le bâton ».

11. «Une activité ou une inhibition est considérée comme volontaire dans la mesure où elle est précédée d'une élaboration mentale anticipatrice $»$ (PIÉRON, 2003).

12. Non sans modifications substantielles (Cf. le chapitre 7 de Pensée et Langage). Le passage d'un plan interne à un plan externe ou le contraire transforment les processus. Une consigne qu'on se donne sans y penser n'est plus la même quand on l'extériorise, à fortiori quand on la formalise non plus pour soi mais pour un soi-disant sosie. Ainsi, malgré des similitudes à certains moments de l'activité, on ne se parle pas à soi-même (activité intrapersonnelle) comme on parle à autrui. Et on ne parle pas à autrui (activité interpersonnelle), comme on se parle à soimême. On ne se parle ni de la même manière ni pour les mêmes raisons.

13. «Les moyens d'agir sur soi ont été initialement des moyens utilisés pour agir sur les autres ainsi que des moyens utilisés par les autres pour agir sur soi», (VYGOTSKI, 2004, p. 233).

14. Le modèle de traitement de l'information de G.A. Miller constitue la référence théorique du travail d'Oddone

15. D'autres règles, également importantes, notamment celles qui concerne le tutoiement et la projection dans le futur ne peuvent pas être examinées dans le cadre de cet article.

16. Du lendemain ou d'un autre jour mais là aussi clairement identifié et spécifié.

17. Pour Aristote, la connaissance procède de l'étonnement. L'étonnement est un instrument de l'élaboration. Si l'« 'extraction' [du savoir implicite] n'est pas finalisée dans la tentative de s'arracher, si peu que ce soit, des énigmes, conflits, empêchements que cette activité-là

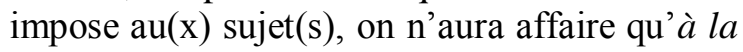
production d'une description de l'activité dont l'énonciation peut rester 'sans surprises' pour le sujet» (Scheller, 2003, p.18, souligné par nous)

18. Ce type d'analyse du travail nécessite le volontariat des participants 
Donner des consignes a un sosie et adopter un autre regard sur les possibilites de developpement des manieres d'agir au travail. Elements de reflexion a partir d'une intervention en sante au travail

19. Dans d'autres cadres, les professionnels transcrivent eux-mêmes le dialogue de l'instruction au sosie et produisent un commentaire écrit. Nous avons cherché dans cet article à identifier les usages de l'analyse $\mathrm{du}$ travail que peuvent faire les professionnels dans le cadre d'un déploiement «allégé » du dispositif.

20. Dans la fonction publique, le rang A correspond aux fonctions d'expertises et d'encadrement. On parle de catégorie $\mathrm{A}^{+}$ (appellation non officielle) pour spécifier que le poste exercé est considéré comme relevant d'un haut niveau de responsabilités C'est le cas de ces directeurs qui ont le grade d'administrateur. Les cadres de formation et les directeurs adjoints dont il sera aussi question sont quant à eux des fonctionnaires de catégorie A.

21. Pour rappel, l'animation d'une réunion d'équipe en particulier. Par exemple, celle de la semaine prochaine, c'est à dire dans le contexte actuel.

22. Le passage du discours à la première personne, forme sociale attendue et spontanée d'une description du travail, au discours à la seconde personne, forme prescrite dans l'exercice ne va pas de soi. Cette dimension, présente à plusieurs endroits du dialogue avec le sosie ne peut pas être analysée dans le cadre de cet article.

23. Destiné dans un second temps à devenir un matériau pour le professionnel.

24. Les questions du sosie ne relèvent pas d'une grille systématique d'entretien. Le recueil de consignes est guidé par l'imagination du psychologue du travail qui se projette dans la situation, se demande quels obstacles il va rencontrer et comment il va les affronter. Même s'il y est préparé, les difficultés à se projeter dans la situation ne sont pas anticipables par le sosie qui s'y confronte au fur et à mesure que le dialogue se déroule. L'adoption de la posture de sosie nécessite un apprentissage rigoureux et une éthique professionnelle.

25. Il s'agit bien sûr pour le psychologue chercheur de construire un cadre de travail qui rend possible ce type de dialogue. L'humour partagé autour de l'incongruité de la situation soutient les efforts des professionnels (24-25).
26. Nous ne disposons pas de ce document

27. Notre transcription «allège» parfois certains énoncés afin de les rendre plus aisément «lisibles ».

28. Cet établissement travaille uniquement avec des formateurs vacataires.

29. Nous reprenons néanmoins la numérotation en cours

30. Piera Aulagnier, citée par Clot, 1999, p.176

31. De la même manière, chaque directeur anime des réunions d'équipe, travaille avec ses adjoints, évalue les collaborateurs etc.

32. La clinique de l'activité partage avec la psychodynamique du travail et les autres approches clinique le projet de "'dépsychologiser' la problématique des risques psychosociaux » (MOLINIER, 2010, p.1999) .

33. La mise en discussion des différences ne va pas de soi d'emblée. Comme l'indique Moscovici invitant le lecteur à se rapporter à sa propre expérience: «On affirme souvent qu'il faut tolérer la différence, ce qui est plus facile à dire qu'à supporter. Sans le vouloir, nous fuyons ce genre de tension et de déplaisir et préférons ne pas nous trouver en face d'un vrai «différend » risquant de les aviver et de les alimenter. Et ce n'est pas sans motif, car la discorde et tout conflit en général sont difficiles à surmonter par les individus, et peut-être même encore davantage par les groupes qui se sentent menacés dans leur existence même » (2000, p.145). Ici, le cadre est conçu pour soutenir l'expression des différences de manière d'agir, qui sont travaillées comme variantes dans l'exercice du métier vs différences interpersonnelles.

34. Une place importante est laissée aux «variantes » soutenue par l'idée que l'activité dépend de la variété des contextes et des objets, de la qualité des instruments dont on dispose ou non, des personnes auxquelles elle s'adresse. La formule «Ça dépend » guide le travail $\mathrm{du}$ groupe.

35. On voudrait signaler ici un problème qu'on n'hésitera pas à qualifier de «redoutable » pour le psychologue du travail. Affirmer les possibilités de développement du pouvoir d'agir et soutenir ces possibilités ne doit en aucun cas occulter la responsabilité de ceux qui dirigent et organisent le travail., qu'il soit 
intervenant ou chercheur. Brigitte Almudever résume ainsi les choses: "Comment [...] affirmer une position qui reconnaît et étudie la part active du sujet dans la construction de son rapport au travail sans pour autant accréditer [...] une déresponsabilisation des organisations quant aux effets pathogènes de certains contextes et modes d'organisations du travail ? » (ALMUDEVER, 2007, p. 196).

36. Mise en tension dont on cherche à ce qu'elle soit créatrice.

\section{Références}

ALMUDEVER, B. Créativité individuelle et collective au travail : enjeux de personnalisation et de changement social. Perspectives en psychologie sociale et du travail. Document de synthèse pour l'Habilitation à diriger des recherches. Toulouse, Université de Toulouse-Le Mirail, 2007

BONNEFOND, J.-Y. Une expérience d'amélioration de la qualité du travail à RenaultFlins. Anact, La revue des conditions de travail, 3, p.66-78, 2015.

BONNEMAIN, A., PERROT, E., \& KOSTULSKI, K. Le processus d'observation, son développement et ses effets dans la méthode des autoconfrontations croisées en clinique de l'activité. Activités, 12-2, 98-124, 2015.

BROSSARD, M. Vygotski. Lectures et perspectives de recherches en éducation. Villeneuve d'Ascq : Presses universitaires du Septentrion, 2004.

CLOT, Y. Le travail sans l'homme ? Pour une psychologie des milieux de travail et de vie. Paris : la Découverte, 1995.

CLOT, Y. La fonction psychologique du travail. Paris : PUF, 1999.

CLOT, Y. Travail et pouvoir d'agir. Paris : PUF, 2008., A., PERROT, E., \& KOSTULSKI,

DEJOURS, C. Travail, usure mentale. De la psychopathologie à la psychodynamique du travail. Paris : Bayard, 1993.

FOLI, O. Emotions et bureaucratie. Frustration intime, paroles de plainte et intégration au milieu. In BERREBI-HOFFMANN, I., Politiques de l'intime. Des utopies sociales d'hier au mondes du travail d'aujourd'hui. Paris : La Découverte, 2009, p. 249-264.

KOSTULSKI, K. Formes et fonctions psychologiques des réalisations langagières. Document de synthèse pour l'Habilitation à diriger des recherches. Paris : Cnam, 2011.

LEONTIEV, A. Activité, conscience, personnalité. Moscou : Editions du Progrès, 1984.

LEONTIEV, A. Le développement du psychisme. Paris : Editions sociales, 1976.

LEPLAT, J. Regards sur l'activité en situation de travail. Paris: PUF, 1997.

LEPLAT, J. Les automatismes dans l'activité : pour une réhabilitation et un bon usage. Activités, 2-2, 43-68, 2005.

MIOSSEC, Y. Les instruments psychosociaux de la santé au travail. Thèse de doctorat de psychologie, CNAM, Paris, 2011.

MIOSSEC, Y., CLOT, Y., \& BOUCHER, C. L'intervention dialogique pour agir sur les risques psychosociaux : une troisième voie. In LEROUGE, L., Approche interdisciplinaire des risques psychosociaux au travail. Toulouse : Octares, 2014, p. 51-66.

MINISTERE DU TRAVAIL. Rapport du Collège d'expertise sur le suivi des risques psychosociaux au travail. Mesurer les facteurs psychosociaux de risque au travail pour les maîtriser, 2011.

MOLINIER, P. Souffrance, défenses, reconnaissance. Le point de vue du travail. Nouvelle revue de psychosociologie, 10, p.99-110, 2010 .

MOSCOVICI, S. Influences conscientes et influences inconscientes. In MOSCOVICI, S. Psychologie sociale des relations à autrui. Paris : Nathan, 2000, p.141-160.

MOSCOVICI, S. L'histoire et l'actualité des représentations sociales. In : MOSCOVICI, S., Le scandale de la pensée sociale. Textes inédits sur les 
Donner des consignes a un sosie et adopter un autre regard sur les possibilites de developpement des manieres d'agir au travail. Elements de reflexion a partir d'une intervention en sante au travail

représentations sociales réunis et préfacés par Nikos Kalampalikis. Paris : Editions de l'EHESS, 2013, p. 65-117

ODDONE, I. (1984). La compétence professionnelle élargie. Société française, 10, 2833.

ODDONE, I., RE, A., \& BRIANTE, G. (1981). Redécouvrir l'expérience ouvrière. Vers une autre psychologie du travail ? Paris : Editions sociales, 1981.

PIERON, H.Volontaire, volonté. In PIERON, H., Vocabulaire de psychologie Paris : PUF, 2003, p.483.

POUZOULET, C. A propos de l'essai de Victor Egger (1881) : «parole intérieure » et formes littéraires du monologue intérieur, 2012. URL: ttp://www.fabula.org/colloques/document1641.php

REILLE-BAUDRIN, E. Reconversion professionnelle, l'espace d'une transition : d'une clinique de l'expérience à l'expérimentation clinique de l'activité transitionnelle : la méthode des instructions au sosie. Thèse de doctorat de psychologie, CNAM, Paris, 2011.

SCHELLER, L. Elaborer l'expérience du travail : activité dialogique et référentielle dans la méthode des instructions au sosie. Thèse de doctorat de psychologie, CNAM, Paris, 2003.
SIMONET, P. L'hypo-socialisation du mouvement : prévention durable des troubles musculosquelettiques chez des fossoyeurs municipaux. Thèse de doctorat de psychologie, CNAM, Paris, 2011.

VYGOTSKI, L. Défectologie et déficience mentale, textes publiés par K. Bariniskov et G. Petitpierre. Lausanne : Delachaux et Niestlé, 1994.

VYGOTSKI, L. Pensée et Langage. Paris : La Dispute, 1997.

VYGOTSKI, L. La signification historique de la crise en psychologie. Lausanne : Delachaux et Niestlé, 1999.

VYGOTSKI, L. Le problème de la conscience dans la psychologie du comportement. In CLOT, Y., Conscience, inconscient, émotions. Paris : La Dispute, 2003, p. 61-94.

VYGOTSKI, L. Psychologie concrète de l'homme. In BROSSARD, M., Vygotski. Lectures et perspectives de recherche en éducation. Villeneuve d'Ascq : Septentrion, 2004, p. 231-255.

\section{Sobre o autor}

Yvon Miossec é psicólogo e doutor em Psicologia do Trabalho. Professor do Conservatoire National des Arts et Métiers, membro da equipe da Clínica da Atividade.

Recebido em setembro de 2017.

Aprovado em outubro de 2017. 